\title{
Combined fluorescent and interferometric detection of protein on a BioCD
}

\author{
Xuefeng Wang, Ming Zhao, and D. D. Nolte* \\ Department of Physics, Purdue University, 525 Northwestern Avenue, West Lafayette, Indiana 47907-2036, USA \\ ${ }^{*}$ Corresponding author: nolte @ physics.purdue.edu
}

Received 20 February 2008; accepted 28 March 2008;

posted 17 April 2008 (Doc. ID 92875); published 13 May 2008

\begin{abstract}
We perform simultaneous interferometric and fluorescent detection of molecular protein layers on a BioCD. The $488 \mathrm{~nm}$ excitation wavelength of fluorescein also provides the interferometric detection channel that operates in a common-path in-line configuration in the condition of phase quadrature set by a thermal oxide on silicon. The simultaneous acquisition of both channels enables a direct correlation between bound mass and fluorescent surface density, which we compare in forward- and reverse-phase immunoassays. Scaling mass sensitivities for immunoassays measured in the interferometric and fluorescent channels are $15 \mathrm{pg} / \mathrm{mm}$ and $1.5 \mathrm{pg} / \mathrm{mm}$, respectively, when applied to gel-printed periodic antibody patterns detected in the frequency domain from the spinning disc. These sensitivities are limited by the inhomogeneities of the print. While fluorescence is subject to bleaching, the interferometry signal is robust under long-term laser illumination. (C) 2008 Optical Society of America

OCIS codes: $\quad 170.2520,170.1470,120.3180,310.6860$.
\end{abstract}

\section{Introduction and Background}

A current direction in assay technology is the development of label-free approaches that use direct detection without the need for fluorescent tags. Surface plasmon resonance has been the leader in this field for many years $[1,2]$, but technologies are emerging that have equal or better sensitivity, and that are more easily multiplexed for high-throughput applications. One of these highly-multiplexed and sensitive direct-detection techniques is the BioCD [3-7]. The BioCD uses spinning-disc interferometry to detect proteins bound out of sample solutions. The BioCD enhances signal-to-noise by spinning and sampling at high-speed to suppress $1 / f$ noise $[8,9]$ rather than boosting signal through resonance effects. The reliance on nonresonant detection allows large format arrays (discs) to be fabricated with a large spot density for high-level multiplexing. However, a disadvantage in the use of interferometry for direct detection is the sensitivity of the BioCD to all bound surface mass whether specific or nonspecific.

0003-6935/08/152779-11\$15.00/0

(C) 2008 Optical Society of America
Fluorescence, on the other hand, is the gold standard of microarray assays [10-12]. The vast majority of commercial assay systems use fluorescence as the detection mechanism. Fluorescence readers are welldeveloped providing high-speed scanning of large array formats, and the signal-processing of microarray data is advanced and sophisticated with solid understanding of systematics to allow their postprocessing compensation [13]. Nonetheless the primary disadvantage of fluorescence detection is the requirement of the fluorescent label. In the case of highly multiplexed assays, this leads to either large numbers of secondary reagents for sandwich assays or to postdevelopment chemistry that can mask the primary binding event. In addition even small molecular tags can alter biological function [14].

For these reasons a dual-mode system that uses fluorescence and interferometry simultaneously takes advantage of the strengths of each to separate out contributions to the assay binding that are the specific interactions separate from nonspecific effects. A key question is what is necessary to perform both interferometry and fluorescence optimally under the same excitation condition. We answer this question through the use of dielectric layers that set the 
appropriate electric field boundary conditions for both modes. It has become well-established that a stand off layer on a reflecting substrate provides an enhancement of fluorescent flux caused by constructive interference of the incident and reflected excitation wave. When the field maximum position coincides with the location of the molecular layer, the fluorescent excitation and emission are both nearly maximized [15]. This effect has been used to detect virus particles [16], thicknesses of bilayers [17], enhanced fluorescence detection on DNA microarrays [18], and molecular interferometry on Bragg stack BioCD platforms [19].

We combine fluorescence detection with label-free interferometry in a single BioCD system scanning proteins on a thermal oxide on silicon. Only a single excitation laser is needed as the source serving both the fluorescence and the interferometric channels. The channels are spatially separated by their different emission anisotropies to provide for sufficient suppression of channel crosstalk. The theory of dual fluorescence and interferometry optimization is discussed in Section 2 followed by the design of the optical system and the silicon disc dielectric structure in Section 3. The system is calibrated using protein stripes printed with a gel-stamp method described in Section 4. Forward- and reverse-phase assays are demonstrated in Section 5 with single-spot sensitivities below $10 \mathrm{ng} / \mathrm{ml}$ in both the interferometric and the fluorescent channels.

\section{Dual Fluorescence and Interferometry Optimization}

To optimize the simultaneous detection of both interferometry and fluorescence requires appropriate surface boundary conditions and electric field magnitudes at the surface where the molecular layer resides. The BioCD operates in reflection and hence causes a standing wave pattern as the incident and reflected waves interfere. Both fluorophore excitation and the optical phase shift from a molecular layer on a surface are maximized when the electric field at the location of the biolayer is a maximum. This establishes an antinode condition of the electric field at the surface as the desired field condition to produce the largest absorption for the fluorophore and the largest optical phase delay of the reflected wave.

The emission wavelength of fluorescence has a Stoke's shift relative to the excitation wavelength, and this wavelength should also have a large electric field amplitude at the surface for enhanced coupling of the fluorescent photons into the far-field. A single dielectric layer on the surface of the substrate can optimize the electric fields for both the excitation and emission wavelengths when the Stoke's shift is not too large (typically $30 \mathrm{~nm}$ ). When chosen appropriately both the excitation and the emission fields can be at or near the field maximum condition.

While both fluorescence and interferometry are optimized by an antinode condition at the surface, there is fundamental difference between the fluorescent and interferometric detection conditions. Fluores- cence is background free in the absence of autofluorescence from the target. Therefore the noise in the fluorescence detection is primarily random additive noise from the detector and electronics. The best signal-to-noise for fluorescence is achieved for an antinode condition with a reflectance of unity to provide the highest photon flux. Interferometry, on the other hand, is not background free. Interferometry relies on a high-intensity reference wave (known as the local oscillator) that has relative intensity noise, which is a random noise proportional to the detected intensity. For the in-line condition the dielectric layer causes the reflection of a stable reference wave off the lower boundary to enable the common-path interferometry. The best signal-to-noise for interferometry is achieved for an antinode condition with a small reflectance (to reduce the relative intensity noise of the reflected wave). This essential incompatibility between fluorescence and interferometry requires a trade-off in the system design. The best compromise for the joint performance of fluorescence and interferometry is a reflectance near $50 \%$. This yields high fluorescence photon flux while providing conditions that maximize the absolute reflectance change, $\Delta R$, caused by a molecular layer on the substrate surface.

Interferometric detection of protein on a BioCD has two orthogonal detection channels. These channels are called differential phase-contrast (DPC) and inline (IL) [19], and they have different optimized conditions. $\mathrm{D} \overline{\mathrm{PC}}$ is a maximum under the condition of a surface field antinode and hence is directly compatible with fluorescence (but requires a smaller reflectance than fluorescence). However, DPC is not the most suitable detection mode when the BioCD carries protein spots. In this case the IL detection mode provides better protein detection performance. The field for optimum IL detection is not at an exact antinode but is displaced to a condition near phase quadrature under purely imaginary $(\pi / 2$ phase) surface reflectance coefficients. However, the quadrature conditions remain near the antinode condition and hence are still approximately compatible with fluorescence.

To describe the field conditions for the DPC and IL modes, we use $\delta_{0, p}$ to denote the phase change in the overlying medium or the protein layer, respectively,

$$
\delta_{0, p}=\frac{2 \pi n_{0, p} \cos \theta_{0, p}}{\lambda} d .
$$

Before the addition of the protein layer, the reflection coefficient is $r$, and after the addition it is $r^{\prime}$. The new Fresnel reflection coefficient, $r^{\prime}$, is related to the original substrate coefficient, $r$, through the expression

$$
r^{\prime}=\frac{\left(e^{i \delta_{p}}-e^{-i \delta_{p}}\right) r_{p}+r\left(e^{-i \delta_{p}}-r_{p}^{2} e^{i \delta_{p}}\right)}{\left(e^{i \delta_{p}}-r_{p}^{2} e^{-i \delta_{p}}\right)+r\left(e^{-i \delta_{p}}-e^{i \delta_{p}}\right) r_{p}} e^{2 i i_{\tan \theta_{p}} \delta_{p}},
$$

where $r^{\prime}$ is determined only by $r$ and $\delta_{p}$ and has no explicit dependence on the details of the substrate structure. The information about the protein layer thickness is contained entirely in $\delta_{p}$. 

give

For a thin molecular layer, Eq. (2) is expanded to

$$
r^{\prime}=r+i P(r) \delta_{p}
$$

where

$$
P(r)=2 \frac{\left(r_{p}-r\right)\left(1-r r_{p}\right)}{\left(1-r_{p}^{2}\right)}+2 r\left(\frac{\tan \theta_{p}}{\tan \theta_{0}}\right) .
$$

Equation (3) approximates a reference wave reflected with the original reflection coefficient from the bare substrate and added to a signal wave with a phase that is linearly dependent on the phase induced by the protein layer. For a typical reflectivity of protein on a homogeneous dielectric support, the function $P(r)$ is mostly real. If $r$ is purely real and positive, the protein produces net phase modulation when the two waves are combined in the far-field. If $r$ is purely imaginary, the protein produces net intensity modulation when the two waves are combined in the far-field. In the general case of $r$, neither purely real nor imaginary, both effects occur together. The detection of net phase modulation in the far-field is achieved using an asymmetric detector such as a split detector with an inverting amplifier [20]. The detection of net intensity modulation in the far-field is achieved using a symmetric detector that simply detects the intensity.

Interferometric detection of a protein layer of thickness $h(x)$ is based on both interference and diffraction upon reflection from the substrate. The twodimensional (2D) diffraction problem is calculated in the Fraunhofer regime. For a Gaussian beam, $g(x, y)$, the reflected near-field is

$$
\begin{aligned}
E(x, y) & =\left[r+i P(r) \delta_{p}\right] g(x, y) \\
& =r[1+i \varphi(r) h(x-v t, y)] g(x, y)
\end{aligned}
$$

where

$$
\begin{aligned}
\phi(r) & =\frac{P(r)}{r} \frac{2 \pi n_{p} \cos \theta_{p}}{\lambda} \\
& =\left[\frac{\left(r_{p}-r\right)\left(1-r r_{p}\right)}{r\left(1-r_{p}^{2}\right)}+\frac{\tan \theta_{p}}{\tan \theta_{0}}\right] \frac{4 \pi n_{p} \cos \theta_{p}}{\lambda} .
\end{aligned}
$$

The far-field is then

$$
E\left(k_{x}, k_{y}\right)=r\left\{G\left(k_{x}, k_{y}\right)+i \varphi(r) \mathrm{FT}[g(x, y) h(x+\eta, y)]\right\},
$$

where FT denotes the Fourier transform and $G$ is the Fourier transform of $g$. The limiting behaviors for the phase in Eq. (6) for nodal and antinodal surfaces are

$$
\phi(r)=\left\{\begin{array}{cc}
0 & \text { nodal } \\
\frac{4 \pi\left(1-n_{p}^{2}\right)}{\lambda} & \text { anti-nodal }
\end{array}\right.
$$

with general substrates having values between these extremes.

The intensity at the detection (Fourier) plane is

$$
\begin{aligned}
I\left(k_{x}, k_{y} ; \eta\right)= & \mid r\left\{G\left(k_{x}, k_{y}\right)+i \phi(r) \mathrm{FT}[g(x, y)\right. \\
& \times h(x+\eta, y)]\}\left.\right|^{2} \approx|r|^{2}\left\{\left|G\left(k_{x}, k_{y}\right)\right|^{2}\right. \\
& -2 G\left(k_{x}, k_{y}\right) \operatorname{Im}[\mathrm{FT}(\phi(r) \\
& \times g(x, y) h(x+\eta, y))]\} .
\end{aligned}
$$

The detected photocurrent is obtained by removing the DC component and integrating Eq. (9) over the Fourier-plane detector response function, $R\left(k_{x}, k_{y}\right)$, that is controlled by appropriate apertures or split detectors. The normalized photocurrent is

$$
\begin{aligned}
i_{d}(\eta)= & \int_{-\infty}^{\infty} R\left(k_{x}, k_{y}\right) I\left(k_{x}, k_{y}, \eta\right) d^{2} k \\
& =-2|r|^{2} \int_{-\infty}^{\infty} R\left(k_{x}\right) G\left(k_{x}\right) \operatorname{Im} \\
& {[\phi \mathrm{FT}(g(x) h(x+\eta))] \mathrm{d} k_{x}, }
\end{aligned}
$$

where the second line is obtained by restricting the problem to the one-dimensional case. The IL signal is acquired by detecting directly all the reflected probe light, while the DPC signal is obtained using a split detector with inversion and summing circuits. The detector response function is

$$
R\left(k_{x}\right)=\left\{\begin{array}{cc}
1 & \text { For IL channel } \\
\operatorname{sgn}(x) & \text { For DPC channel }
\end{array}\right.
$$

and the current is

$$
\begin{aligned}
& i_{d}(\eta)= \\
& \quad-2|r|^{2} \operatorname{Im}\left\{\phi \int_{-\infty}^{\infty} R\left(k_{x}\right) G\left(k_{x}\right) \mathrm{FT}[g(x) h(x+\eta)] \mathrm{d} k_{x}\right\} .
\end{aligned}
$$

The two different detector output currents are proportional to the quantities

$$
\begin{aligned}
& i_{d}^{\mathrm{IL}}(\eta)=-2|r|^{2} \operatorname{Im}\left\{\left.\phi[g(x) \otimes(g(x) \cdot h(x+\eta))]\right|_{x=0}\right\}, \\
& i_{d}^{\mathrm{DPC}}(\eta)=-2|r|^{2} \operatorname{Im}\left\{\left.i \phi[d(x) \otimes(g(x) \cdot h(x+\eta))]\right|_{x=0}\right\},
\end{aligned}
$$

where $d(x)$ is the Dawson function (the Hilbert transformation of $g(x)$, which is an asymmetric function). The IL and DPC currents are

$$
\begin{aligned}
& i^{\mathrm{IL}}(x)=-2 \phi_{\operatorname{Im}}|r|^{2}\left[g^{2}(x) \otimes h(x)\right], \\
& \quad i^{\mathrm{DPC}}(x)=-2 \phi_{\operatorname{Re}}|r|^{2}[(d(x) \cdot g(x)) \otimes h(x)],
\end{aligned}
$$

with the phase functions 


$$
\begin{aligned}
\phi_{\mathrm{Re}} & =\frac{4 \pi n_{p} \cos \theta_{p}}{\lambda} \operatorname{Re}\left(\frac{\left(r_{p}-r\right)\left(1-r r_{p}\right)}{r\left(1-r_{p}^{2}\right)}+\frac{\tan \theta_{p}}{\tan \theta_{0}}\right) \\
\phi_{\mathrm{Im}} & =\frac{4 \pi n_{p} \cos \theta_{p}}{\lambda} \operatorname{Im}\left(\frac{\left(r_{p}-r\right)\left(1-r r_{p}\right)}{r\left(1-r_{p}^{2}\right)}\right) .
\end{aligned}
$$

Simplifying the DPC expression gives the final form for the two channels

$$
\begin{aligned}
& i^{\mathrm{IL}}(x)=-2 \phi_{\mathrm{Im}}|r|^{2}\left(g^{2} \otimes h\right), \\
& i^{\mathrm{DPC}}(x)=\phi_{\mathrm{Re}}|r|^{2} \sigma\left[g^{2} \otimes \frac{d h}{d x}\right],
\end{aligned}
$$

where $\sigma$ is the beam radius in the Gaussian beam profile $g$.

The complementary behaviors of the two channels are made clear by the last expressions. DPC senses the differential protein height, being most sensitive to slopes and steps while being insensitive to areas with uniform thickness. The far-field symmetry for DPC is antisymmetric, and the phase-contrast sensitivity is maximized when the reflectivity is real and positive (antinodal surface). The IL channel has the opposite attributes, sensing the direct protein height with a far-field that is symmetric, and the IL channel is maximized with a reflectivity that is purely imaginary. One way to achieve a purely imaginary substrate reflectivity is with an eighth-wavelength dielectric layer on a high-index substrate. Calculations for $30^{\circ}$ and the wavelength is $488 \mathrm{~nm}$, which is the excitation wavelength for FITC show that $\varphi_{\operatorname{Im}}|r|^{2}$ reaches an extrema of \pm 0.0027 (IL channel optimized) when $r= \pm 0.58 i$, and $\varphi_{\operatorname{Re}}|r|^{2}$ reaches an extrema of \pm 0.0272 (DPC channel optimized) when $r=1$ (antinode disc).

\section{Optical System and Data Acquisition}

\section{A. Optical System}

The optical system for dual-channel detection on a spinning disc requires angular separation between the fluorescent signal and the interferometric signal. To achieve this separation, the probe laser is incident on the disc at a finite angle. The interferometric signal is the specular reflection of the probe off the disc surface at the reflection angle, while the fluorescent signal is acquired normal to the surface as shown in Fig. 1. Although normal-incidence operation for both channels could be accomplished using a dichroic beam splitter, the oblique-incidence design acts as a spatial filter that is more convenient than a wavelength filter.

The illumination wavelength from the argon laser is $488 \mathrm{~nm}$ for excitation of the fluorescein isothiocyanate (FITC), $\mathrm{C}_{21} \mathrm{H}_{11} \mathrm{NO}_{5} \mathrm{~S}$ with a molecular weight of 389.38 , fluorophore with emission at $510 \mathrm{~nm}$. The $40 \mathrm{~mW}$ laser beam is focused onto the BioCD surface through a $10 \mathrm{~cm}$ convex lens. The radius of the focal spot is $\sim 18 \mu \mathrm{m}$ on the disc surface. Higher resolution can be achieved by switching the $10 \mathrm{~cm}$ lens with a short focal-length lens or a microscope objective lens. The specular interferometric signal is detected by a silicon photodiode through a $488 \mathrm{~nm}$ bandpass filter. The fluorescence signal is detected through a $4 \mathrm{~cm}$ convex lens above the BioCD that focuses the fluorescence onto the avalanche photodiode (APD C5460-01) through two $510 \mathrm{~nm}$ long-pass optical filters that block the scattered $488 \mathrm{~nm}$ light. The signals from the detectors are sent to a digital oscilloscope where they are averaged nine times on a single track (fixed radius) and acquired to a computer. The radius is changed to a new track with a typical pitch of $20 \mu \mathrm{m}$, and the data for the new track is acquired. In this way a $2 \mathrm{D}$ map of the disc surface is constructed in both channels.

The APD has an amplification of $0.05 \times 10^{9} \mathrm{~V} / \mathrm{W}$. The background noise of the APD is $\sim 6 \mathrm{pW}$ with a noise-equivalent power (NEP) of $0.02 \mathrm{pW} / \mathrm{Hz}^{1 / 2}$ and a $100 \mathrm{kHz}$ bandwidth. This detector limit is equivalent to a $0.3 \mathrm{pm}$-thick protein layer conjugated with fluorescein or $\sim 0.4 \mathrm{pg} / \mathrm{mm}^{2}$ protein planar density on the disc. The $100 \mathrm{kHz}$ frequency response of the APD sets the upper limit for the detection speed. If the system scans protein spots with a $100 \mu \mathrm{m}$ diameter on a BioCD with a $0.05 \mathrm{~s}$ spin period $(20 \mathrm{~Hz})$, the central frequency is on the outer ring of the disc, which is within the frequency range of the detector.

To scan a full disc, two free coordinates form a polar coordinate system. The motor produces the angular coordinate and spins in a selectable frequency from $20 \mathrm{~Hz}$ to $80 \mathrm{~Hz}$. A linear stage produces the radial polar coordinate, which can be moved with a $0.1 \mu \mathrm{m}$ linear precison and a $300 \mathrm{~mm}$ maximum travel distance. The motor is fixed on the linear stage to produce 2D maps with appropriate computer control. This system is capable of mapping a $100 \mathrm{~mm}$ diameter BioCD in 30 min with $20 \mu \mathrm{m} \times 20 \mu \mathrm{m}$ pixel resolution. The optical system of Fig. 1 consists of a laser (INNOVA300, Coherent), a linear stage (MM2K, Newport), a motor (scanner motor, Laser Lines), the BioCD, a silicon photodiode detector (PC50-6, Pacific Silicon Sensor), an avalanche photodiode detector (C5460-01, Hamamatsu), an oscilloscope, and a computer.

\section{B. BioCD Disc Structure and Protein Immobilization}

The BioCD uses a silicon wafer coated with a silica thermal oxide [19] $100 \mathrm{~nm}$-thick optimized for interferometry at a wavelength of $488 \mathrm{~nm}$ incident at an angle of $30^{\circ}$. As described above, this structure is also nominal for fluorophore excitation, because the excitation efficiency is proportional to the square of the surface electric field, which is $|1+r|^{2}$, where $r$ is disc reflection coefficient. The reflection coefficient is calculated to be $r=0.267-0.245 i$ under the conditions of the $488 \mathrm{~nm}$ probe wavelength, the $30^{\circ}$ incident angle, the s-polarization, and a $100 \mathrm{~nm}$ silica film on the silicon substrate. Calculation shows that $1 \mathrm{~nm}$ monolayer protein causes $2.2 \%$ reflectance increment if applied on the surface. For $|1+r|^{2}=1.67$, the fluorescence excitation efficiency is improved by $67 \%$ compared with protein on glass, and the fluorescence 


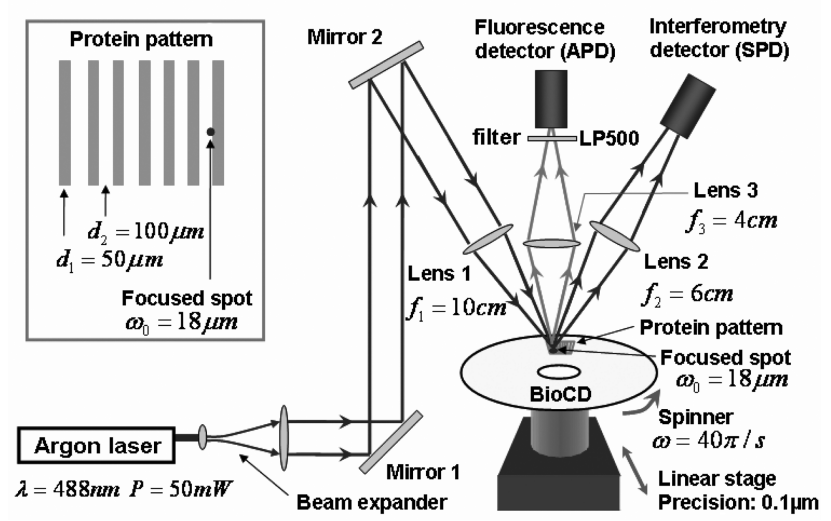

Fig. 1. Experimental layout using the $488 \mathrm{~nm}$ line from an argon laser incident at $30^{\circ}$ and focused on the BioCD. The interferometric signal is detected in the reflection direction, while the fluorescence signal is collected by a lens situated above the disc. The oblique-incidence design spatially separates the two channels.

collection efficiency is also improved by $|r|^{2}=13 \%$ due to reflectance. Hence the disc is balanced for good performance on both the fluorescence and the interferometry channels.

Protein molecules are immobilized on the BioCD with physical adsorption in which hydrophobic activation of the silicon dioxide is performed by surface silanization. Discs are soaked in $0.02 \mathrm{M}$ chlorooctadecylsilane toluene solution for $12 \mathrm{~h}$ followed by washes with toluene, acetone, methanol, and finally deionized water. Proteins bind to the silanized disc surface through hydrophobic interaction.

Two protein patterns are used for this work. The first is a periodic pattern of stripes that are used for frequency-domain acquisition to measure signalto-noise performance and for comparison between the interferometry and fluorescence channels. The grating pattern is printed with a gel-stamp method [19]. Each protein stripe width is $50 \mu \mathrm{m}$ with a $\overline{100} \mu \mathrm{m}$ gap between two stripes $(150 \mu \mathrm{m}$ period). The other consists of discrete hand-pipetted spots with volumes of $0.5 \mu \mathrm{l}$ for each spot. After printing, the disc surface is rinsed with deionized water then blown dry with nitrogen.

\section{Detection of Moving Fluorophores on Surfaces}

Detecting moving fluorescent spots under a stationary excitation beam is fully equivalent to detecting a stationary fluorescent spot with a rastered excitation, an approach that is commonly used in commercial fluorescent scanners. However, the BioCD has the potential to spin at high speed with a raster velocity significantly higher than linear raster scanners. Therefore we estimate the effect of high-speed scanning on fluorescence detection.

A consequence of the disc motion and the finite measurement time is the effective stretching of the Gaussian excitation beam into a stripe oriented in the direction of motion. For a maximum disc linear velocity at the rim of $30 \mathrm{~m} / \mathrm{s}$ for a $100 \mathrm{~Hz}$ spin frequency and a sampling time, $\Delta t$, on the oscilloscope, the profile of the illumination area can be considered as the convolution of Gaussian function with the linear transit distance $v \Delta t$. The oscilloscope recording time for every point is $1 \mu \mathrm{s}$, which is also the avalanche photodiode detector response time. This yields a stretched length of $30 \mu \mathrm{m}$ compared with the beam diameter of $2 w_{0}=36 \mu \mathrm{m}$ defined by $I(r)=\exp \left(-2 r^{2} / w_{o}^{2}\right)$. In addition the protein spoke and spot diameters are $\sim 100 \mu \mathrm{m}$. Therefore the Gaussian beam convolved with both the transit length and the protein length yields a response function with a width of $55 \mu \mathrm{m}$. As a result under these conditions, the $100 \mu \mathrm{m}$ protein width is sampled with a $55 \mu \mathrm{m}$ resolution. Therefore high-speed scanning blurs the image if the acquisition does not have sufficiently high bandwidth. We use a $20 \mathrm{~Hz}$ spin frequency to avoid blurring.

Another effect that could be caused by the high scan speed would occur if the excited state lifetime is long or comparable to the laser transit time. In this case, a fluorophore could move out of the optical collection volume before a photon is emitted. However, typical emission times are on the order of nanoseconds, while transit times are on the order of a microsecond. Therefore this effect is negligible.

To estimate the detected fluorescence flux, the fluorescent conversion is given by

$$
P_{\text {fluo }}=\ln (10) \frac{\lambda_{\text {laser }}}{\lambda_{\text {fluo }}} \kappa \alpha A P_{\text {Laser }},
$$

where $P_{\text {fluo }}$ is the fluorescence photon flux, $A$ is the beam area, $\alpha$ is the absorption coefficient, $k$ is the quantum yield, $\lambda_{\text {fluo }}$ is the emission wavelength, and $\lambda_{\text {laser }}$ is the excitation wavelength. The FITC is conjugated with proteins via the amine group. The absorption coefficient is in the range of $7.69 \times 10^{4}$ to $8.80 \times 10^{4} \mathrm{~L} \mathrm{~cm}^{-1} \mathrm{~mol}^{-1}[19,21,22]$ at $490.5 \mathrm{~nm}$. Each protein molecule is combined with several FITCs. The molecular ratio of fluorescein and protein is 3-5 for the sample used in this work (antirabbit IgG-FITC, F0382, Sigma). The quantum yield for a conjugated FITC is $<0.4$ [23], and the molecular weight of an antibody molecule is $\sim 150 \mathrm{kDa}$. For our excitation beam power and assuming a $1 \mathrm{~nm}$-thick fluoroscene-conjugated molecular layer, the total fluorescence power is $\sim 7.5 \mu \mathrm{W}$. Not all fluorescence reaches the detector. The focal length of the fluorescence collection lens is $4 \mathrm{~cm}$ with a diameter of $2.5 \mathrm{~cm}$. The solid angle formed by this lens and focal spot is $0.8 \mathrm{sr}$. If the fluorescence emits isotropically, the collected fluorescence is $0.8 / 4 \pi=6.4 \%$. Therefore an APD with a dynamic range of $0.1-100 \mathrm{nW}$ is appropriate for the fluorescence detector.

\section{System Performance and Calibration}

\section{A. Fluorointerferometric Correlation of Captured Protein}

We applied the dual-channel BioCD to the detection of protein captured during a reverse-phase immunoassay to test the correlation between the two channels. We printed goat IgG (R2004, Sigma) as the reference 
protein in a periodic pattern with the gel-stamp method, then backfilled the gaps of the gratings with rabbit IgG (I5006, Sigma) as the antigen to capture the antibody from the solution. A prescan and a postscan were performed before and after incubation with concentrations of $10 \mathrm{ng} / \mathrm{ml}$ and $10 \mu \mathrm{g} / \mathrm{ml}$ antirabbit IgGFITC (F0382, Sigma) for $30 \mathrm{~min}$. Figure 2 shows the differences between the prescans and the postscans for the two channels for the two concentrations. The area for the protein pattern is $1 \mathrm{~mm}$ vertical by $4 \mathrm{~mm}$ horizontal $=4 \mathrm{~mm}^{2}$. Both the fluorescence channel and the interferometry channel show clear binding of the fluorescently tagged antibody to the rabbit antigen. By taking the difference of the prescan and the postscan, surface roughness and other static surface topology are mostly removed from the interferometry channel. Residual surface roughness is caused by slight data misregistration between the scans, which contributes to a background signal in the interferometric channel that is absent in the fluorescent channel.

The periodic protein patterns in Fig. 2 are analyzed in the frequency domain as power spectra in Fig. 3. Figure 3(a) shows the power spectra for the interferometry channel for the two concentrations, and Fig. 3(b) shows the power spectra for the corresponding fluorescence channel. The two spectra in each figure are referenced to the high-frequency noise floors at $500 \mathrm{kHz}$ for the respective channels, while the zero $\mathrm{dB}$ level is set at the peak of the first spatial harmonic of the protein pattern. This allows a direct comparison of the signal-to-noise between the two concentrations within one channel and for a comparison of the performance between the interferometric and fluorescence
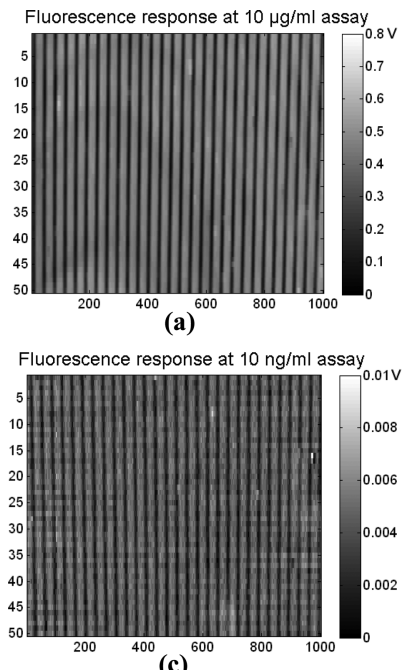

(c)
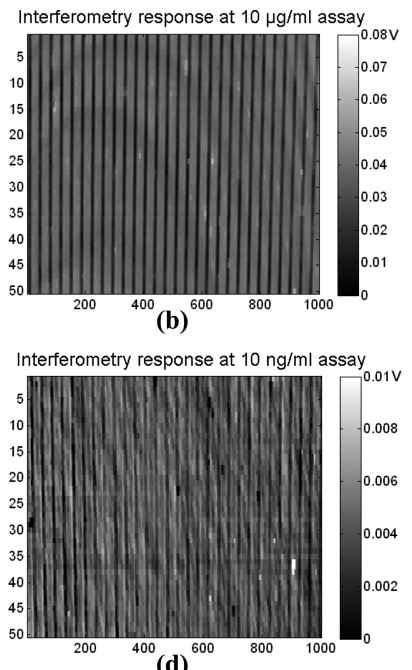

(d)
Fig. 2. Reverse-phase immunoassay performed on backfilled antigen in the fluorescence and interferometry channels. The discs were printed with reference protein goat IgG using a gel stamp then backfilled with rabbit IgG. The areas were incubated with $10 \mu \mathrm{g} / \mathrm{ml}$ or $10 \mathrm{ng} / \mathrm{ml}$ antirabbit IgG-FITC. (a) Fluorescence response in reverse assay $(10 \mu \mathrm{g} / \mathrm{ml})$. (b) Interferometry response in reverse assay $(10 \mu \mathrm{g} / \mathrm{ml})$. (c) Fluorescence response in reverse assay $(10 \mathrm{ng} / \mathrm{ml})$. (d) Interferometry response in reverse assay $(10 \mathrm{ng} / \mathrm{ml})$. channels. The first harmonic in the data is the fundamental periodicity of the protein pattern. The approximately square-wave pattern produces the higher harmonics. These harmonic peaks ride on a broad shoulder that decreases with increasing frequency to a noise floor approximately $-120 \mathrm{~dB}$ for both channels at high frequency (above $300 \mathrm{kHz}$ ). The broadfrequency shoulder is not random noise, but arises from the actual structures on the disc. These arise from the inhomogeneities in the printed patterns (for both channels) and from disc roughness (in the interferometric channel). For the $10 \mu \mathrm{g} / \mathrm{ml}$ data, the signalto-background ratio of the interferometric channel is 300:1, while for the fluorescence channel is 1000:1. The difference between these two ratios is due to the presence of surface roughness in the interferometry channel, and its absence in the fluorescence channel. The $10 \mathrm{ng} / \mathrm{ml}$ data show signal-to-noise ratios of 10:1 and 100:1 for interferometry and fluorescence, respectively.

From the signal-to-noise ratios, it is possible to estimate the detection sensitivities of the two channels.
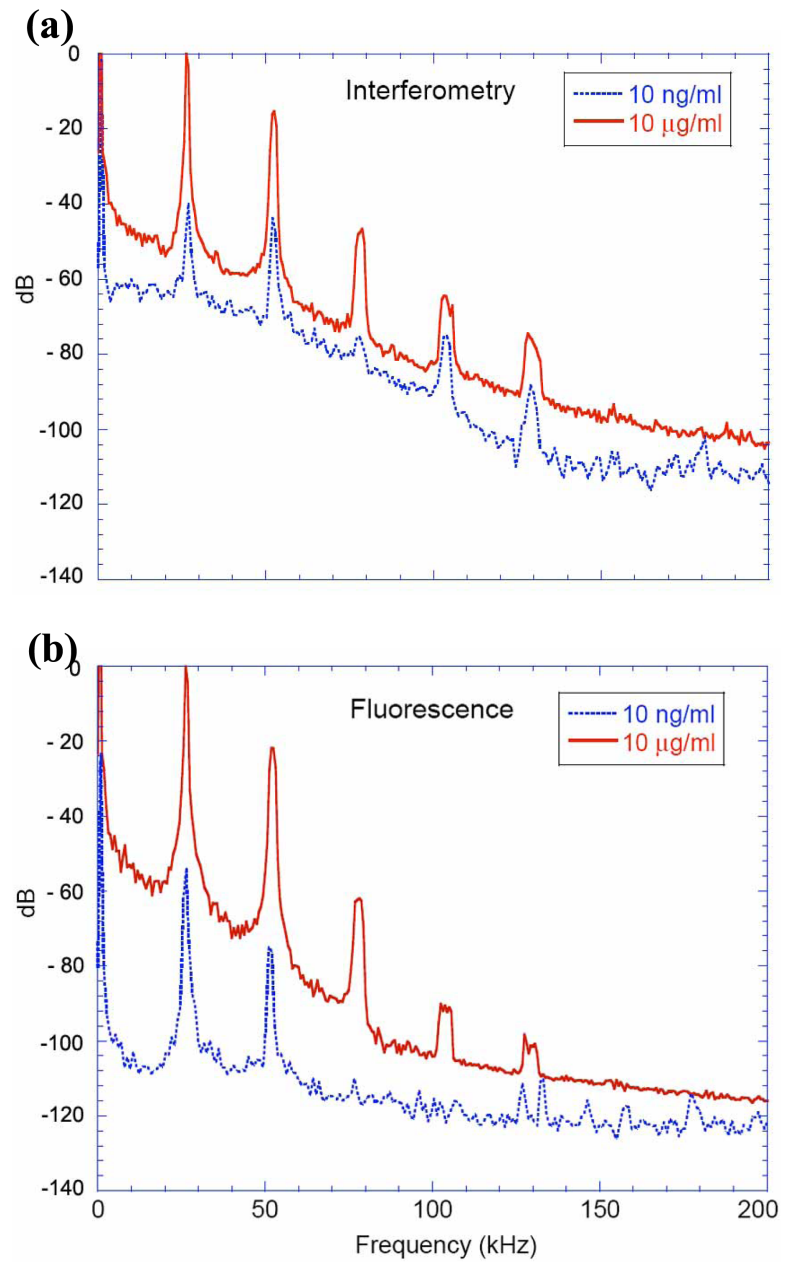

Fig. 3. (Color online) Power spectra for (a) interferometric and (b) fluorescent responses of the periodic protein patterns of Fig. 2 from both channels at $10 \mu \mathrm{g} / \mathrm{ml}$ and $10 \mathrm{ng} / \mathrm{ml}$ incubation concentrations. The graphs are normalized by the $10 \mu \mathrm{g} / \mathrm{ml}$ first harmonic and related to the high-frequency noise floor. 
For the $10 \mathrm{ng} / \mathrm{ml}$ data, the average protein height is $0.4 \mathrm{~nm}$ using the conversion of Eq. (12). The height resolution is set as the protein height divided by the signal-to-noise ratio. This gives a minimum detectable protein height of $40 \mathrm{pm}$ for the IL interferometry and $4 \mathrm{pm}$ for fluorescence.

Mass per area as is not an appropriate unit for the evaluation of the sensitivity of a mass-sensitive biosensor because averaging over larger chip areas yields a smaller standard error, which improves the detection limit. This leads to the situation that chips with different areas have different detection limits for protein. Therefore mass per area is an extrinsic property and it is not the valid quantity for comparisons among different detection systems that average over different areas. The appropriate scaling quantity that does allow comparisons among different experiments is the scaling mass sensitivity defined by

$$
S_{\text {scal }}=\rho \Delta h \sqrt{\text { Detection Area }},
$$

where $\Delta h$ is the minimum surface height measured in the detection area. For the $10 \mathrm{ng} / \mathrm{ml}$ data in Fig. 2(b), $\Delta h$ is calculated to be $40 \mathrm{pm}$ for the IL interferometry and $4 \mathrm{pm}$ for fluorescence based on one track scan. The area of the track is approximately $36 \mu \mathrm{m} \times 4 \mathrm{~mm}=$ $0.144 \mathrm{~mm}^{2}$, which yields scaling surface mass sensitivities of $\mathrm{S}_{\mathrm{scal}}^{\mathrm{IL}}=15 \mathrm{pg} / \mathrm{mm}$ and $\mathrm{S}_{\mathrm{scal}}^{\mathrm{FL}}=1.5 \mathrm{pg} / \mathrm{mm}$ for the interferometric and fluorescent (FL) channels, respectively. These sensitivities are for immunoassays and are limited by the inhomogeneities in the gel printing process and the antigen capture. Fundamental metrology limits can be much lower [24].

An important aspect of the data is the correlation between the interferometric and fluorescent channels. In principle these channels may measure different effects with interferometry measuring all mass changes, whether fluorescent or not. The correlation between the two channels is plotted in Fig. 4(a) for $10 \mu \mathrm{g} / \mathrm{ml}$ and in Fig. $4(\mathrm{~b})$ for $10 \mathrm{ng} / \mathrm{ml}$. The data were selected from Fig. 2 from the center of the bright and dark stripes. Data from the edges of the stripes were not plotted to avoid image registration and edge effects. The two protein levels are clearly separated at $10 \mathrm{ng} / \mathrm{ml}$. The correlations are nearly linear in each case, with correlation coefficients of 0.92 and 0.47 for $10 \mu \mathrm{g} / \mathrm{ml}$ and $10 \mathrm{ng} / \mathrm{ml}$, respectively. The coefficients of variation (CV) for the backfilled area at $10 \mu \mathrm{g} / \mathrm{ml}$ were $5.5 \%$ and $4 \%$ for the interferometric and fluorescent channels and at $10 \mathrm{ng} / \mathrm{ml}$ were $39 \%$ and $11 \%$, respectively. In the $10 \mathrm{ng} / \mathrm{ml}$ case, interferometry has a larger CV than fluorescence because of the higher background that is measured in the interferometric channel. The CVs at the higher concentration, on the other hand, are nearly equal between the interferometric and fluorescent channels.

\section{B. Bleaching Study}

A disadvantage of fluorescent techniques is that fluorophores are prone to bleaching. Interferometry, on the other hand, is immune to bleaching and is therefore
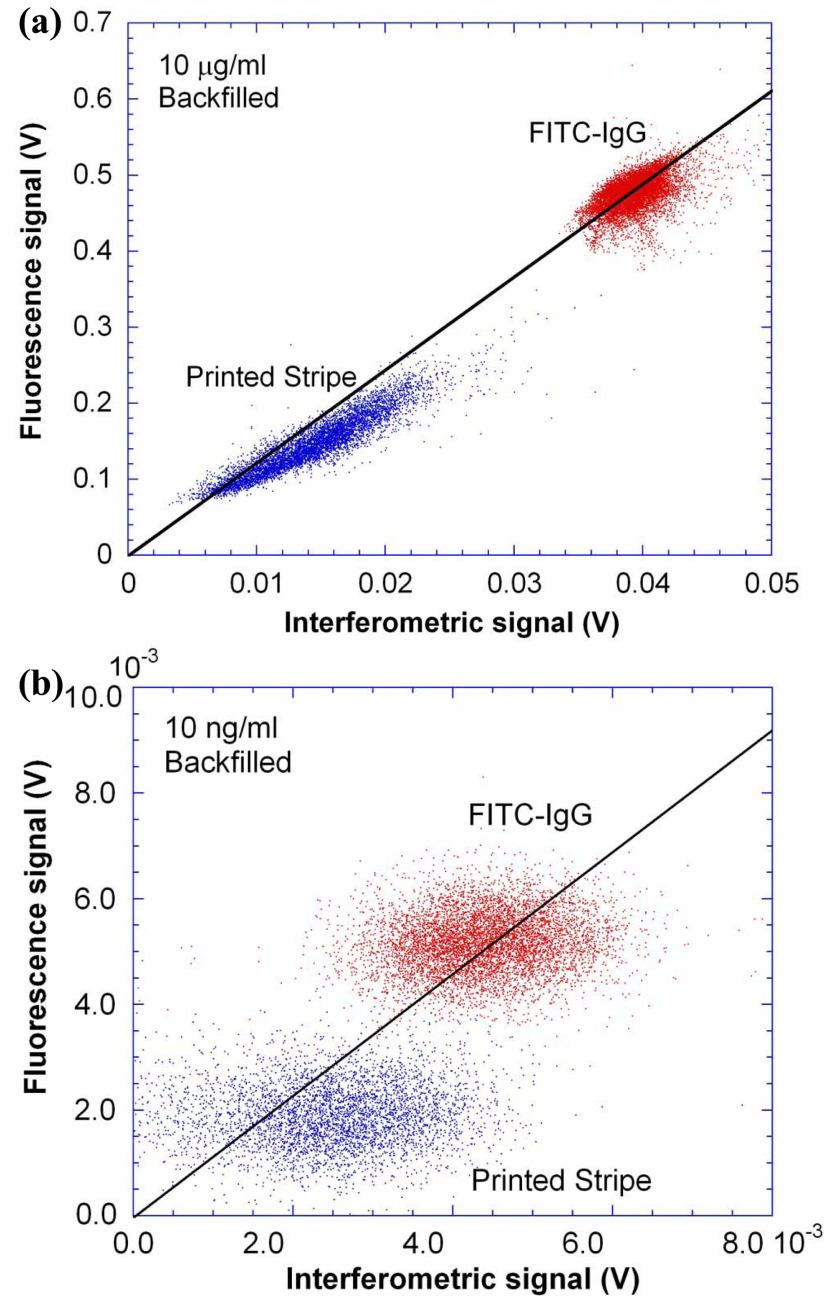

Fig. 4. (Color online) Scatter plots between the interferometric and fluorescent responses at the incubation concentration of (a) $10 \mu \mathrm{g} / \mathrm{ml}$ and (b) $10 \mathrm{ng} / \mathrm{ml}$ in Fig. 2 .

more stable and quantitative. We performed an experiment to measure the time-course response on a reverse-phase assay in the fluorescence and interferometry channels. The preparation of the sample was the same as that in Subsection 4.B. One track of the sample was scanned every $6 \mathrm{~s}$. The protein on this track was illuminated by the focused laser with $40 \mathrm{~mW}$ power. The disc spun at a $20 \mathrm{~Hz}$ frequency, the radius of the focal spot was $18 \mu \mathrm{m}$, and the detection radius was $40 \mathrm{~mm}$. The average power flux density on the protein is estimated as $40 \mathrm{~mW} /$ $(2 \pi \cdot 40 \mathrm{~mm} \cdot 36 \mu \mathrm{m})=4.5 \mathrm{~mW} / \mathrm{mm}^{2}$. This is the average value over time, equivalent to $40 \mathrm{~mW}$ distributed over a ring at the detection radius. The instantaneous density is $4 \mathrm{~kW} / \mathrm{mm}^{2}$.

Figures 5(a) and 5(b) show the time-course response (the difference between the postscan and the prescan) of the fluorescent and interferometric channels, respectively. Figure 5(c) shows the signal variation of the two channels as a function of time. The bleaching data are fit with a biexponential curve with two bleaching lifetimes. Although there is only one type of fluorophore in the sample, the fluoro- 
phore may have two bleaching pathways or two environments $[25,26]$. The curve fit yields $T_{1}=70$ and $T_{2}=478$ in the fluorescence channel while being immeasurably large for the interferometry channel. One conclusion of this experiment is that bleaching of fluorophores does not modify the refractive index by an observable amount at $\lambda=488 \mathrm{~nm}$. The fraction of FITC by weight is $\sim 0.2 \%$. Therefore bleaching of FITC in this experiment would not be expected to be measurable in the interferometry channel.

\section{Immunoassays}

\section{A. Sandwich Assay}

To test the sensitivities of protein detection in a highbackground concentration in the fluorescent and interferometric channels, we adopted a sandwich assay approach. We immobilized the antibody on the disc, then incubated the antibody with the corresponding analyte solution (antigen) in a high protein background. The captured antigen was incubated with the secondary antibody conjugated with fluorescein. The fluorescent intensity and the interferometric signal increment depend on the antigen concentration in the original solution. This experiment produces a response curve for the antigen concentration.

In the experimental procedure, eight wells of antibody spots were printed on the BioCD. Each well consisted of a $2 \times 2$ array of spots. Two diagonal spots (upper-left and lower-right) were antirabbit IgG (R2004, Sigma), the other two spots used antimouse IgG (M8642, Sigma) as the control. These eight wells were incubated with $0,0.01,0.03,0.1,0.3,1,3$ and $10 \mu \mathrm{g} / \mathrm{ml}$ rabbit IgG (I5006, Sigma) in $7 \mathrm{mg} / \mathrm{ml}$ bovine serum. The disc was scanned again after incubation. All spots were incubated with $20 \mu \mathrm{g} / \mathrm{ml}$ antirabbit biotin (B8895, Sigma), $20 \mu \mathrm{g} / \mathrm{ml}$ avidin (A9275, Sigma), and $20 \mu \mathrm{g} / \mathrm{ml}$ antiavidin (F1269, Sigma) in sequence. All incubation times were $30 \mathrm{~min}$. Scanning was performed after each incubation.

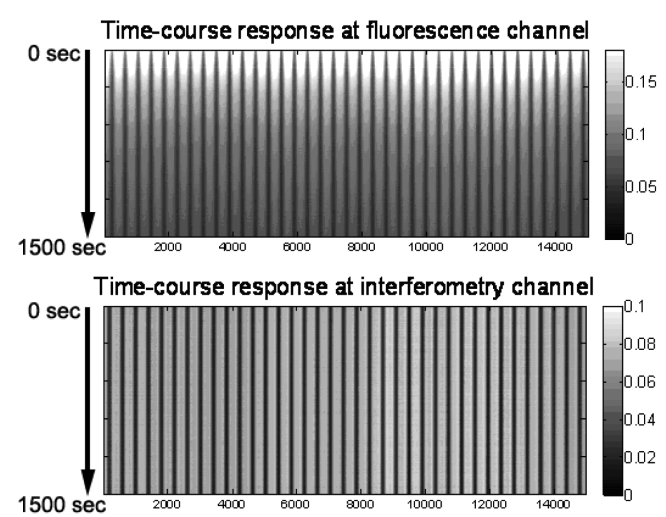

(a)
The top four panels in Fig. 6 show the images of the protein spots from the interferometric channel. Scan 1 shows the thickness of the spots after incubation with the series of rabbit IgG concentrations (labelfree forward-phase). Scan 2 shows the thickness of the spots after incubation with biotinylated antirabbit. Scan 3 shows the thickness of the spots after incubation with avidin. Scan 4 shows the thickness of the spots after incubation with antiavidin FITC. The fifth line shows fluorescence signals after incubation with antiavidin FITC.

The response curves in Fig. 7 show the thickness increments caused by each incubation. In the three interferometric curves, the response data increase monotonically with analyte concentration. The continuous curves are nominal fits to the data using a 4-parameter response curve based on a stretched Langmuir function. The lowest interferometric curve is the forward assay (immobilized antibody binding antigen from solution) and is label free. The second curve is the nonfluorescent sandwich using a biotinylated antibody. The third interferometric curve is the combined binding of avidin and the secondary fluorescent antiavidin antibody. The fluorescence curve corresponds to the third interferometric curve, showing good agreement between the interferometric and fluorescent channels from the final scan. Both channels reach a $10 \mathrm{ng} / \mathrm{ml}$ detection limit in a sandwich assay in the presence of a $7 \mathrm{mg} / \mathrm{ml}$ protein background for the sandwich assay. The label-free step in the interferometry channel has a detection limit of $\sim 30 \mathrm{ng} / \mathrm{ml}$.

\section{B. Simultaneous Forward- and Reverse-Phase Assays}

Forward- and reverse-phase assays can both be applied to the detection of protein concentrations. The reverse-phase assay has a higher sensitivity because one antigen molecule can bind with several antibody molecules, providing an amplification that enhances the sensitivity of the reverse assay. On the

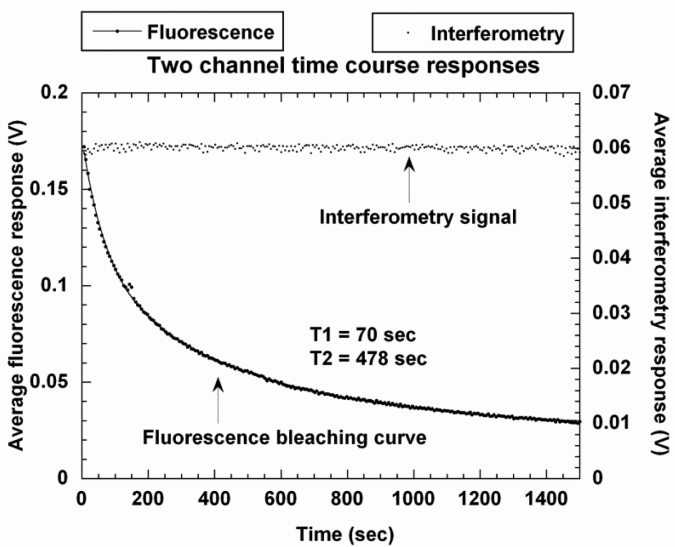

(b)

Fig. 5. Two-channel scans performed continuously on the same track consisting of antibody conjugated with fluorescein after a reversephase assay. The time-course scanning results on (a) the fluorescence channel and (b) the interferometry channel as a function of position and time. The fluorescence becomes weaker with time (increasing downward) due to bleaching. (c) Signal intensity variation of the two channels as a function of time. 


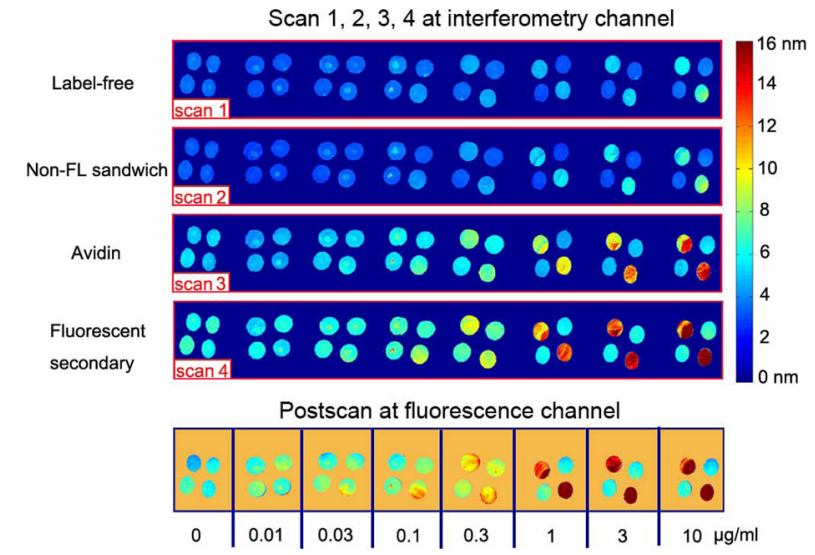

Fig. 6. (Color online) Sandwich assay of rabbit IgG. The panels are after printing antirabbit IgG (Scan 1), incubation with 0 , $0.01,0.03,0.1,0.3,1,3$, and $10 \mu \mathrm{g} / \mathrm{ml}$ rabbit IgG, respectively, in $7 \mathrm{mg} / \mathrm{ml}$ rat lysate (Scan 2 ), and incubation with $20 \mu \mathrm{g} / \mathrm{ml}$ antirabbit IgG-biotin (Scan 3). The fluorescence scan is acquired after incubation with the antiavidin FITC.

other hand, this amplification effect is not likely to manifest itself at a low analyte concentration. Therefore at low concentrations the reverse assay may not be superior to the forward assay. We compare the simultaneous performance of both a forward- and a reverse-phase assay at analyte concentrations ranging from $30 \mathrm{ng} / \mathrm{ml}$ to $30 \mu \mathrm{g} / \mathrm{ml}$.

We printed eight wells of rabbit IgG and antigoat IgG on the BioCD with $2 \times 2$ spots in one well (see Fig. 8, first row). In each well, the upper-left and lower-right spots are antigoat IgG spots, and the other two are rabbit IgG spots. The analyte for this experiment is antirabbit IgG-FITC cultured in goat (F0382, Sigma). The analyte binds with rabbit IgG (I5006, Sigma) as the antibody in a forward-phase reaction, while it can simultaneously bind with antigoat IgG (G6638, Sigma) as the antigen in a reverse-phase reaction. In this way the forward and reverse assays

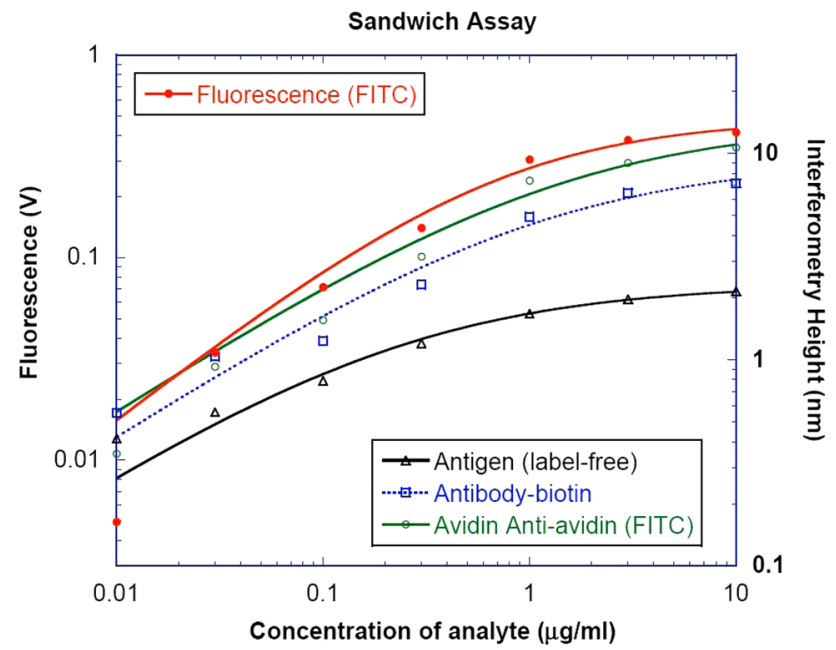

Fig. 7. (Color online) Concentration response curves for Fig. $\underline{6}$ fitted with four-parameter Langmuir functions. The detection limits of both channels reach $10 \mathrm{ng} / \mathrm{ml}$ in a $7 \mathrm{mg} / \mathrm{ml}$ protein background. are tested in the same well at the same time. The eight wells were incubated with concentrations of $0,0.03,0.1,0.3,1,3,10$, and $30 \mu \mathrm{g} / \mathrm{ml}$ of the goat antirabbit IgG. Two-channel scans were performed before and after incubation. The IL and FL responses are shown in Fig. 8 in the third and forth panels.

The concentration response curves for the simultaneous forward-and reverse-phase assays are shown in Fig. 9. The interferometric responses are solid data, while the fluorescent responses are open data. The forward assays are marked by squares and the reverse by circles. The forward assays agree well between the fluorescent and the interferometric channels, exhibiting similar Langmuir behavior. The reverse assays are notably different than the forward assays, showing much larger response, and exhibiting different functional dependences between the fluorescent and the interferometric channels. The large response of the reverse assays above $1 \mu \mathrm{g} / \mathrm{ml}$ is expected to be caused by partial cross-linking of the polyclonal antibodies by dissociated antigen from the disc surface. At low concentrations (below $1 \mu \mathrm{g} / \mathrm{ml}$ ), the response of the forward assay is slightly larger than for the reverse assay, suggesting that the reverse assay has no amplification relative to the forward assay at low concentrations.

\section{Discussion and Prospects}

The complexity of surface chemistry in the performance of solid-phase immunoassays can be understood by using complementary detection channels that acquire information simultaneously from the same location on the chip. For instance, nonspecific binding and residues accompany the binding of specific target analytes to recognition molecules immobilized on the chip surface. Fluorescence techniques have been valuable in discriminating between these contributions through the use of a secondary specific molecule conjugated with a fluorophore. However, in

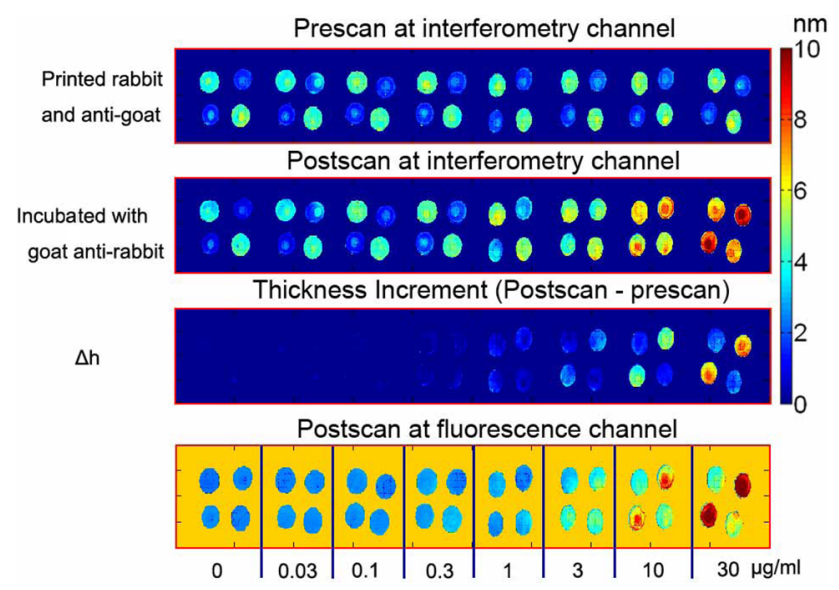

Fig. 8. (Color online) Simultaneous forward- and reverse-phase assays compared in the two channels. In each well, upper-left to lower-right, spots are antigoat IgG spots, while the other two spots are rabbit IgG antigen spots. The eight wells are incubated with 0 , $0.03,0.1,0.3,1,3,10$, and $30 \mu \mathrm{g} / \mathrm{ml}$ antirabbit-FITC IgG cultured in goat, respectively. 


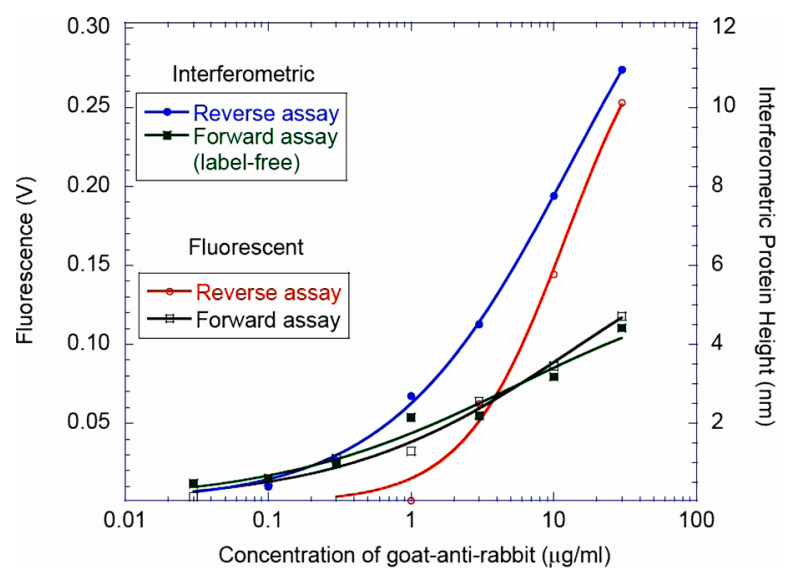

Fig. 9. (Color online) The concentration response curves for Fig. 8 fitted with four-parameter Langmuir functions. In both channels the reverse-phase assay has stronger responses than the forwardphase assay when the incubation concentration is above $3 \mu \mathrm{g} / \mathrm{ml}$, whereas the forward-phase assay is stronger when the incubation concentration is below $1 \mu \mathrm{g} / \mathrm{ml}$

the new era of highly multiplexed detection, the use of many secondary labels becomes prohibitive, and label-free detection modes are actively being sought. These are often mass-sensitive detection schemes that detect all bound mass, specific or not.

Therefore we find ourselves in a transitional period as conventional fluorescent techniques yield to new label-free techniques such as evanescent wave sensors and molecular interferometry. During this transitional period, the use of hybrid systems that continue to operate fluorescently while simultaneously operating in a label-free mode will provide important cross-validation and cross-correlation, comparing the new to the established performances.

We have demonstrated such a dual-channel system that acquires fluorescent data simultaneously and colocationally with molecular interferometric data from a BioCD. The BioCD is an ideal platform to perform this hybrid fluorescent-interferometric detection, because the two channels share the same excitation laser, and both channels benefit from the high-speed access to broad areas (lending itself to high multiplexing) and high-speed sampling that reduces the data acquisition noise. Fast fluorophore lifetimes remain compatible with high-speed scanning and hence cause no reduction in fluoroescent photon detection efficiency for fluorescent detection. In addition the same dielectric substrate structure, in this case a thermal oxide on silicon, can be near-optimal for both the fluorescent and the interferometric channels.

Strong correlation between the fluorescent and interferoemtric channels was observed for periodic gelprinted protein patterns on the disc detected in the frequency domain. At high concentrations the two channels share similar statistical variations, while at lower concentrations $(10 \mathrm{ng} / \mathrm{ml})$ the presence of nonfluorescent bound mass does limit the interferomeric detection channel. Scaling mass sensitivities for the interferometric and fluorescent channels were $15 \mathrm{pg} / \mathrm{mm}$ and $1.5 \mathrm{pg} / \mathrm{mm}$, respectively, for reverse-phase antibody capture. To relate these scaling values with conventional sensitivities expressed in units of mass per area (used by other mass detection approaches), they are divided by the square root of the area over which the measurement is averaged. Quite simply, if the measurement area is $1 \mathrm{~mm}^{2}$, the conventional mass sensitivities are $15 \mathrm{pg} / \mathrm{mm}^{2}$ and $1.5 \mathrm{pg} / \mathrm{mm}^{2}$ for the interferometric and fluorescent channels, respectively.

As an example of the value of dual-channel detection, we studied the binding properties of forward- and reverse-phase immunoassays performed simultaneously with an analyte that bound either as antigen or as antibody to immobilized species on the BioCD. We were able to show that at lower concentrations, the forward-and reverse-phase binding were comparable, while at high concentrations (above $1 \mu \mathrm{g} / \mathrm{ml}$ ) the reverse-phase binding exhibits an amplification over the forward-phase binding. It is likely this is caused by the ability of affinity-purified polyclonal antibodies to bind to more than one epitope.

In conclusion we have performed simultaneous interferometric and fluorescent detection of molecular protein layers on a BioCD. The $488 \mathrm{~nm}$ wavelength excitation of fluorescein also provides the interferometric detection channel that operates in a common-path IL configuration in the condition of phase quadrature set by a thermal oxide on silicon. The simultaneous acquisition of both channels enables a direct correlation between bound mass and fluorescent dipole density, which we have compared in forward- and reverse-phase immunoassays applied to gel-printed periodic antibody patterns detected in the frequency domain.

This work was sponsored under grants from Quadraspec Incorporated and from the Indiana Economic Development Corporation through the Purdue Research Foundation.

\section{References}

1. J. Homola, S. Yee, and G. Gauglitz, "Surface plasmon resonance sensors: review," Sens. Actuators B Chem. 54, 315 (1999).

2. C. Boozer, G. Kim, S. X. Cong, H. W. Guan, and T. Londergan, "Looking towards label-free biomolecular interaction analysis in a high-throughput format: a review of new surface plasmon resonance technologies," Curr. Opin. Biotechnol. 17, 400405 (2006).

3. L. Peng, M. M. Varma, F. E. Regnier, and D. D. Nolte, "Adaptive optical biocompact disk for molecular recognition," Appl. Phys. Lett. 86, 183902-1-3 (2005).

4. L. Peng, M. M. Varma, W. Cho, F. E. Regnier, and D. D. Nolte, "Adaptive interferometry of protein on a BioCD," Appl. Opt. 46, 5384-5395 (2007).

5. M. M. Varma and D. D. Nolte, "Spinning-disk self-referencing interferometry of antigen-antibody recognition," Opt. Lett. 29, 950-952 (2004).

6. M. M. Varma, H. D. Inerowicz, F. E. Regnier, and D. D. Nolte, "High-speed label-free detection by spinning-disk microinterferometry," Biosens. Bioelectron. 19, 1371-1376 (2004). 
7. M. Zhao, L. L. Peng, W. Cho, F. Regnier, and D. D. Nolte, "Phase contrast BioCD: high-speed immunoassays at subpicogram detection levels," Proc. SPIE-Int. Soc. Opt. Eng. 6095, L950 (2006).

8. M. Zhao, X. Wang, and D. Nolte, "The in-line-quadrature BioCD," Proc. SPIE-Int. Soc. Opt. Eng. 6447, B4470 (2007).

9. M. Zhao, D. D. Nolte, W. R. Cho, F. Regnier, M. Varma, G. Lawrence, and J. Pasqua, "High-speed interferometric detection of label-free immunoassays on the biological compact disc," J. Clin. Chem. Clin. Biochem. 52, 2135-2140 (2006).

10. M. F. Templin, D. Stoll, M. Schrenk, P. C. Traub, C. F. Vohringer, and T. O. Joos, "Protein microarray technology," Trends Biotechnol. 20, 160-166 (2002).

11. S. Nagl, M. Schaeferling, and O. S. Wolfbeis, "Fluorescence analysis in microarray technology," Microchimica Acta 151, 1-21 (2005).

12. H. G. Park, J. Y. Song, K. H. Park, and M. H. Kim, "Fluorescence-based assay formats and signal amplification strategies for DNA microarray analysis," Chem. Eng. Sci. 61, 954965 (2006).

13. M. Schena, D. Shalon, R. W. Davis, and P. O. Brown, "Quantitative monitoring of gene-expression patterns with a complementary-DNA microarray," Science 270, 467-470 (1995).

14. H. H. Szeto, P. W. Schiller, K. S. Zhao, and G. X. Luo, "Fluorescent dyes alter intracellular targeting and function of cellpenetrating tetrapeptides," FASEB J. 18, 118-120 (2004).

15. M. Bras, V. Dugas, F. Bessueille, J. P. Cloarec, J. R. Martin, M. Cabrera, J. P. Chauvet, E. Souteyard, and M. Garrigues, "Optimisation of a silicon/silicon dioxide substrate for a fluorescence DNA microarray," Biosens. Bioelectron. 20, 796 (2004).

16. H. Ewers, V. Jacobsen, E. Klotzsch, A. E. Smith, A. Helenius, and V. Sandoghdar, "Label-free optical detection and tracking of single virions bound to their receptors in supported membrane bilayers," Nano Lett. 7, 2263-2266 (2007).
17. V. Kiessling and L. K. Tamm, "Measuring distances in supported bilayers by fluorescence interference-contrast microscopy: polymer supports and SNARE proteins," Biophys. J. 84, 408-418 (2003).

18. H. Choumane, N. Ha, C. Nelep, A. Chardon, G. O. Reymond, C. Goutel, G. Cerovic, F. Vallet, C. Weisbuch, and H. Benisty, "Double interference fluorescence enhancement from reflective slides: application to bicolor microarrays," Appl. Phys. Lett. 87, 031102 (2005).

19. X. F. Wang, M. Zhao, and D. D. Nolte, "Common-path interferometric detection of protein monolayer on the BioCD," Appl. Opt. 46, 7836-7849 (2007).

20. M. Zhao, W. R. Cho, F. Regnier, and D. Nolte, "Differential phase-contrast BioCD biosensor,” Appl. Opt. 46, 61966209 (2007).

21. H. Leonhard, L. Gordon, and R. Livingst, "Acid-base equilibria of fluorescein and 2', 7'-dichlorofluorescein in their ground and fluorescent states," J. Phys. Chem. 75, 245-249 (1971).

22. P. C. DeRose and G. W. Kramer, "Bias in the absorption coefficient determination of a fluorescent dye, standard reference material 1932 fluorescein solution,” J. Lumin. 113, 314320 (2005)

23. G. P. Derbalian, N. Kameda, and G. L. Rowley, "Fluorescein labeling of fab while preserving single thiol," Anal. Biochem. 173, 59-63 (1988).

24. D. D. Nolte and M. Zhao, "Scaling mass sensitivity of the BioCD at $0.25 \mathrm{pg} / \mathrm{mm}$," Proc. SPIE-Int. Soc. Opt. Eng. 6380, U127-U132 (2006).

25. E. K. L. Yeow, S. M. Melnikov, T. D. M. Bell, F. C. De Schryver, and J. Hofkens, "Characterizing the fluorescence intermittency and photobleaching kinetics of dye molecules immobilized on a glass surface," J. Phys. Chem. A 110, 1726-1734 (2006).

26. M. Prummer and M. Weiss, "Bulk fluorescence measurements cannot probe the survival-time distribution of single molecules," Phys. Rev. E 74, 021115 (2006). 